\title{
A new biomarker in patients with vitiligo: a case- control study
}

\begin{abstract}
Background: CRP is an acute phase protein secreted in the blood stream by the liver in response to inflammatory cytokines such as IL6 and several other systemic inflammation biomarkers. Since inflammatory and immune factors have a key role in the pathogenesis of vitiligo, we aimed to assess the relationship between the serum level of hsCRP (as a biomarker for systemic inflammation) and the pathogenesis of vitiligo.
\end{abstract}

Methods: In this case-control study, we enrolled patients who had referred to the Dermatology Department of Valiasr Dermatology Hospital, Birjand, Iran, during 2013. The patients were diagnosed to have vitiligo by a dermatologist. The patients were divided into two groups: those with type A vitiligo (generalized, $n=30$ ) and those with type $B$ vitiligo (segmental, $n=30$ ). Moreover, 30 people who had the inclusion criteria and did not have vitiligo were selected among those referring to the clinic as the control group and matched with the other two groups. The serum hsCRP levels were checked for all the patients in the three groups and compared. Data were compared between the three groups and analyzed using SPSS software.

Results: We did not find any significant difference between the three groups with respect to sex, age, smoking habit, BMI, and history of diabetes mellitus. The serum level of hsCRP was $4.76 \pm 1.31 \mathrm{mg} / 1$ in patients with type a vitiligo, $3.71 \pm 1.03$ in those with type B vitiligo, and $3.01 \pm 1.08$ in those in the control group. The highest level of serum hsCRP was related to patients with type A vitiligo while the lowest mean was related to the control group. The mean serum hsCRP level was significantly higher in patients with type A compared with those with type $\mathrm{B}$ and the control group $(\mathrm{P}<0.001)$. However, in patients with type $\mathrm{B}$ and the control groups no significant different was seen in this regard $(\mathrm{P}=0.053)$. Moreover, we found that the serum level of hsCRP did not differ between the three groups with respect to BMI.

Conclusion: We found an association between increased serum hsCRP level and generalized vitiligo. The usage of hsCRP alone is neither sensitive, nor specific in diagnosis of vitiligo but this association could imply that hsCRP could intensify the severity of vitiligo thus serum hsCRP level might be useful for evaluating the disease activity of vitiligo as Novel biomarker.

Keywords: Biomarker; Vitiligo; HsCRP; Vitellius; Autoimmunity
Volume 3 Issue 6 - 2016

Reza Ghaderi, ${ }^{1,2}$ Pouya Nezafati ${ }^{3}$

'Department of Dermatology, Birjand University of Medical Sciences, Iran

${ }^{2}$ Medical Toxicology Research Center, Mashhad University of Medical Sciences, Iran

${ }^{3}$ Student Research Committee, Mashhad University of Medical Sciences, Iran

Correspondence: Reza Ghaderi, Department of Dermatology, Faculty of Medicine, Birjand University of Medical Sciences, Moallem St, Birjand, Iran, Medical Toxicology Research Center, Mashhad University of Medical Sciences, Mashhad, Iran, Tel 98563244300I, Email Rezaghaderi@yahoo.com

Received: July 30, 2016 | Published: August 22, 2016

\section{Introduction}

The word vitiligo is possibly derived from "Vitellius" meaning white leather patches. ${ }^{1}$ This disease is acquired lack of pigmentation following the absence of epidermal melanocytes. ${ }^{1}$ The destruction of melanocytes is the result of depigmented macules clinically seen in this disease. Although its cause is unknown, several theories have been presented such as autoimmunity, autocytotoxicity, oxidative stress and neuro-hormonal complications, ${ }^{2-4}$ the most common of which is autoimmunity with a genetic background. ${ }^{3}$ Vitiligo often begins in childhood or young adulthood. ${ }^{2}$ Almost half of the affected individual sure initially afflicted before the age of 20 and its prevalence decreases with age. Its global prevalence has been estimated to be $1-2 \% .{ }^{2,4}$ It equally affects men and women and there is no association between skin type and vitiligo. ${ }^{3}$

Vitiligo presents in the form of hypo pigmented macules or white patches with varying degrees of depigmentation. Vililigo is classified based on the extent of involvement and the distribution of depigmentation. ${ }^{2}$ The most common manifestation of vitiligo is the generalized type in the form of bilateral symmetrical depigmentations on the face, neck, extensor surfaces or bony surfaces on the hands, feet, wrists, axilla and mucosal surfaces. ${ }^{1,3}$ The acrofacial type occurs on the fingers and periorificial areas with circular patterns. The focal type presents as focal depigmented macules without any dermatological pattern. The segmental types has asymmetrical dermatological pattern accompanied by autoimmune diseases. In the universal type, hypopigmentation is seen in all body surfaces. ${ }^{2,4}$ In most patients, the depigmentation begins in areas exposed to sunlight. ${ }^{2}$

Vitiligo increases the risk of autoimmune diseases such as Hashimoto's thyroiditis and Graves disease, Addison's disease, pernicious anemia, diabetes type 1, psoriasis, uveitis, candidiasis, polyglandular autoimmune syndromes, and alopecia areata. ${ }^{1,4}$ Its diagnosis is clinical and biopsy is rarely needed. However, histopathology is helpful in diagnosis and shows the absence of melanocytes and infiltration of inflammatory sells. In order to determine the extent and activity of vitiligo, Wood's light examination is required. ${ }^{3}$

The pathogenesis of vitiligo is associated with the destruction of melanocytes resulting from immune and inflammatory mediators. ${ }^{5}$ The most important theory about the pathogenesis of vitiligo is the autoimmune theory in which cytokines and inflammatory mediators such as GM-CSF, TNF $\alpha$, IL6, IL1, and IL8 play a key role. ${ }^{6}$ 
Various studies have shown the relationship between cytokines and depigmentation in vitiligo. Il6 facilitates reactions between melanocytes and leukocytes and IL8 induces neutrophil and polymorpho nuclear chemo toxicity at inflammation site. Difference studies have shown the increased expression of pre-inflammatory cytokines such as IL6 and TNF $\alpha$ with inhibitory effects on pigmentation in vitiligo.

Hypersensitive C-reactive protein (hsCRP) test is a quantitative laboratory test that analyzes very low amounts of CRP in the serum. This test is commonly used as a diagnostic and prognostic marker for cardiac diseases and is a comprehensive index for evaluating coronary complications. CRP is an acute phase protein secreted in the blood stream by the liver in response to inflammatory cytokines such as IL6 and several other systemic inflammation biomarkers. ${ }^{8-10}$

Standard CRP (normal levels of 0-0.5 mg/l) has been used for years in patients with acute inflammation or for evaluating evident chronic inflammation. A level of less than $1 \mathrm{mg} / \mathrm{l}$ is considered low risk, 1-3 $\mathrm{mg} / \mathrm{l}$ medium risk, and more than $3 \mathrm{mg} / \mathrm{l}$ as high risk inflammation. For levels more than $10 \mathrm{mg} / \mathrm{l}$ the source of inflammation or infection should be sought and the test should be repeated after recovery. ${ }^{11}$

HsCRP has also been used as an acceptable index for milder systemic inflammation in several disorders such as diabetes as well as cardiac and chronic obstructive pulmonary diseases. ${ }^{12}$ Since inflammatory and immune factors have a key role in the pathogenesis of vitiligo and as the cause of this disease is still unknown and any reliable clue will be considered valuable, we aimed to assess the relationship between the serum level of hsCRP (as a marker for systemic inflammation) and the pathogenesis of vitiligo.

\section{Patients and methods}

In this case-control study, we enrolled patients who had referred to the Dermatology Department of Valiasr Dermatology Hospital, Birjand, Iran, during 2013. The patients were diagnosed to have vitiligo by a dermatologist. The inclusion criteria were BMI 1830 (based on the formula $\mathrm{kg}$ weight divided by square height in meters), no history of using anti-inflammatory drugs, corticosteroids and statins, and hormone therapy. We excluded patients who were pregnant, had infections or inflammatory diseases such as rheumatoid arthritis, psoriasis, and other diseases of the connective tissues, as well as those who had specific underlying diseases such as coronary artery disease, atherosclerosis, metabolic disease, and chronic pulmonary disease. Oral informed consent was obtained from the participants. This study was performed with the approval of the ethics committee at Birjand University of Medical Sciences.

The patients were divided into two groups: those with type A vitiligo (generalized, $\mathrm{n}=30$ ) and those with type $\mathrm{B}$ vitiligo (segmental, $\mathrm{n}=30$ ). The patients were matched with respect to age, sex, smoking habit, and diabetes mellitus. Moreover, 30 people who had the inclusion criteria and did not have vitiligo were selected among those referring to the clinic as the control group and matched with the other two groups. The serum hsCRP levels were checked for all the patients in the three groups. The patients should have fasted for 8 hours and must not have used the drugs mentioned in the exclusion criteria one month prior to the test. In the laboratory, $3 \mathrm{~mL}$ blood samples were taken from the patients and were transferred to the laboratory after clotting. After centrifugation (DBC kit, Canada), the serum hsCRP level was measured. Data were compared between the three groups and analyzed using SPSS software, version 20, Fisher Exact Test.

Kolmogorov-Smirnov test was used to check normal distribution. If data were distributed normally, one-way analysis of variance
(ANOVA) was used. If data were not distributed normally, KruskalWallis and Mann-Whitney test were used as appropriated. $\mathrm{P}$ value $<0.05$ was considered as statistically significant.

\section{Results}

The mean age of the participants in the case group was $48.18 \pm 16.29$ years. The mean age of the participants in the control group was $47.83 \pm 13.79$ years. $17(56.7 \%), 19(63.3 \%)$, and $16(53.3 \%)$ of the patients with type A, type B, and those in the control group were men, respectively (Table 1 ). The mean height of the patients with type A, type B, and those in the control group was $169.7,168.9$ and $168.9 \mathrm{~cm}$. The mean \pm SD BMIs of the patients with vitiligo type A, type B, and the control group were $24.09 \pm 2.42,24.67 \pm 2.85$, and $24.28 \pm 1.18 \mathrm{~kg} /$ $\mathrm{m}^{2}$, respectively (Table 2). The BMIs of $19(63.3 \%), 15(50 \%)$, and $21(70 \%)$ of the patients with type A, type B, and those in the control group were in the normal range, respectively. $4(13.3 \%), 3(10 \%)$, and $4(13.3 \%)$ of the patients with type A, type B, and those in the control group were smokers, respectively (Table 3$) .3(10 \%), 2$ (6.7\%), and $3(10 \%)$ of the patients with type A, type B, and those in the control group had diabetes mellitus, respectively (Table 4). We did not find any significant difference between the three groups with respect to sex, age, smoking habit, BMI, and history of diabetes mellitus.

The serum level of hsCRP was $4.76 \pm 1.31 \mathrm{mg} / \mathrm{l}$ in patients with type A vitiligo, $3.71 \pm 1.03$ in those with type $B$ vitiligo, and $3.01 \pm 1.08$ in those in the control group (Table 5). The highest level of serum hsCRP was related to patients with type A vitiligo while the lowest mean was related to the control group. In the patients with type A vitiligo the highest level of serum hsCRP was $7.28 \mathrm{mg} / \mathrm{l}$ and the lowest 2.53 $\mathrm{mg} / \mathrm{l}$. In the patients with type B vitiligo the highest level of serum hsCRP was $5.31 \mathrm{mg} / \mathrm{l}$ and the lowest $1.83 \mathrm{mg} / \mathrm{l}$. In the control group the highest level of serum hsCRP was $5.16 \mathrm{mg} / \mathrm{l}$ and the lowest 1.17 $\mathrm{mg} / \mathrm{l}$ (Table 6).

Tukey's post hoc test results showed that the mean serum hsCRP level was significantly higher in patients with type A compared with those with type $\mathrm{B}$ and the control group $(\mathrm{P}<0.001)$. However, in patients with type $\mathrm{B}$ and the control groups no significant different was seen in this regard $(\mathrm{P}=0.053)$. The mean serum hsCRP levels were higher in men in general, as well as the men in the two case groups. However, in the control group the serum hsCRP level was in the same range. ANOVA test was used to compare serum hsCRP levels in the three groups. The results showed that the difference was significant between the groups, but not between the sexes and no interaction was seen in this regard. The mean hsCRP level was higher in patients with normal BMI compared to those that were overweight. Moreover, we found that the serum level of hsCRP did not differ between the three groups with respect to BMI.

\section{Discussion}

The highest level of serum hsCRP was related to patients with type a vitiligo while the lowest mean was related to the control group. We found that the mean serum hsCRP level was significantly higher in patients with type a compared with those with type $\mathrm{B}$ and the control group. Moreover, we found that the serum level of hsCRP did not differ between the three groups with respect to sex and BMI. In a previous study, the researchers found that type A and B vitiligo have different pathogenesis and the autoimmune theory is only applicable to type $\mathrm{a}^{13}$ which is consistent with our findings.

We found that $13.3 \%, 10 \%$, and $13.3 \%$ of the patients with type a, type B, and those in the control group were smokers. Since smoking 
affects the serum hsCRP level, it was considered as a confounding factor in this study and the groups were matched in this regard.

In our study, the serum hsCRP level was $4.99 \mathrm{mg} / \mathrm{l} \mathrm{n}$ the men and $4.46 \mathrm{mg} / \mathrm{l}$ in the women with type A vitiligo. The corresponding figures were 3.83 and $3.51 \mathrm{mg} / \mathrm{l}$, respectively, in those with type B vitiligo and 3 and $3.03 \mathrm{mg} / \mathrm{l}$ in the control group. To the best of our knowledge, we did not find any similar study comparing generalized

Table I Distribution of subjects based on sex in different groups (type A) and segmental (type B) vitiligo with respect to serum hsCRP levels. Therefore, we could not compare our findings with other study. However, there were several studies regarding hsCRP levels in other diseases. One study evaluated serum hsCRP levels in 51 patients with psoriasis and compared them with a control group, showing no significant relationship. ${ }^{14}$ The mean serum hsCRP level was 5 $\mathrm{mg} / \mathrm{l}$ in 146 patients with rheumatoid arthritis, showing systemic inflammation. ${ }^{15}$

\begin{tabular}{llll}
\hline Groups & Gender & & Total N (\%) \\
\cline { 2 - 3 } & Female N (\%) & Male N (\%) & $100(30)$ \\
Vitiligo Type A & $13(43.3)$ & $17(56.7)$ & $100(30)$ \\
Vitiligo Type B & $11(36.7)$ & $19(63.3)$ & $100(30)$ \\
Control & $14(46.7)$ & $16(53.3)$ & $100(90)$ \\
Total & $38(42.2)$ & $52(57.8)$ & \\
X2=0.64 df=2 p=0.73 & & & \\
\hline
\end{tabular}

Table 2 Distribution of subjects based on BMI in different groups

\begin{tabular}{llll}
\hline Groups & $\begin{array}{l}\text { BMI } \\
\text { Normal } \\
\text { N (\%) }\end{array}$ & $\begin{array}{l}\text { Overweight } \\
\text { N (\%) }\end{array}$ & $\begin{array}{l}\text { Total } \\
\text { N (\%) }\end{array}$ \\
\hline Vitiligo Type A & II (36.7) & $19(63.3)$ & $100(30)$ \\
Vitiligo Type B & $15(50)$ & $15(50)$ & $100(30)$ \\
Control & $9(30)$ & $21(70)$ & $100(30)$ \\
All & $35(38.9)$ & $55(61.1)$ & $100(90)$ \\
Fisher Exact Test=2.57 p=0.32 & & &
\end{tabular}

Table 3 Distribution of subjects based on smoking in different groups

\begin{tabular}{llll}
\hline Groups & Smoking & Total N (\%) \\
\cline { 2 - 4 } & Yes & No & $100(30)$ \\
Vitiligo Type A & $4(I 3.3)$ & $26(86.7)$ & $100(30)$ \\
Vitiligo Type B & $3(I 0)$ & $27(90)$ & $100(30)$ \\
Control & $4(I 3.3)$ & $26(86.7)$ & $100(90)$ \\
All & II $(I 2.2)$ & $79(87.8)$ & \\
Fisher Exact Test=0.3I p=I & & & \\
\hline
\end{tabular}

Table 4 Distribution of subjects based on DM in different groups

\begin{tabular}{llll}
\hline Groups & Yes N (\%) & No N (\%) & Total \\
\hline Vitiligo Type A & $3(10)$ & $27(90)$ & $100(30)$ \\
Vitiligo Type B & $2(6.7)$ & $28(93.3)$ & $100(30)$ \\
Control & $3(10)$ & $27(90)$ & $100(30)$ \\
All & $8(8.9)$ & $82(91.1)$ & $100(90)$ \\
Fisher Exact Test=0.4I p=I & & & \\
\hline
\end{tabular}

Table 5 Comparison of HSCRP serum level in groups

\begin{tabular}{|c|c|c|c|c|c|}
\hline Variable & Mean & Standard Deviation & $\mathbf{F}$ & df & $\mathbf{p}$ \\
\hline Vitiligo Type A & 4.76 & 1.31 & & & \\
\hline Vitiligo Type B & 3.71 & 1.03 & & & \\
\hline Control & 3.01 & 1.08 & 17.62 & (87 & $<0.001$ \\
\hline
\end{tabular}

Table 6 Level of HSCRP in different groups

\begin{tabular}{|c|c|c|c|c|c|c|c|}
\hline Variable & Mean & $\begin{array}{l}\text { Standard } \\
\text { Deviation }\end{array}$ & Variance & Median & Mode & Minimum & Maximum \\
\hline Type A Vitiligo & 4.75 & $\mid .3 \mathrm{I}$ & 1.72 & 4.8 & 3.56 & 2.53 & 7.28 \\
\hline Type B Vitiligo & 3.71 & 1.03 & 1.05 & 3.68 & 4.25 & 1.83 & 5.31 \\
\hline Control & 3.01 & 1.08 & 1.17 & 2.87 & 4.18 & 1.17 & 5.16 \\
\hline
\end{tabular}

In another study on patients with non-segmental vitiligo, the researchers found that the serum level of IL6 and GM-CSF was higher in patients with focal and generalized vitiligo compared to the control group, while Il-1B was only higher in patients with generalized vitiligo. Since these markers are inflammatory markers, it can be stated that inflammation has a key role in the pathogenesis of vitiligo, 
especially generalized vitiligo, and hsCRP is an inflammatory marker that increases in systemic inflammation. ${ }^{6}$ Moreover, the serum level of hsp-70 (an acute phase protein) increases significantly in patients with vitiligo. ${ }^{13}$

Based on our knowledge, this is the first study to investigate the association between serum hsCRP level and vitiligo but recently, studies were performed to investigate serum hsCRP levels in other autoimmune and inflammatory diseases. ${ }^{16}$ Recently Lochhead, et al., ${ }^{17}$ found out Plasma levels of interleukin 6 and high-sensitivity C-reactive protein before diagnosis are associated with risk of incident inflammatory bowel disease. ${ }^{17}$ In another study Botta et al showed patients with active rheumatoid arthritis had elevated hsCRP levels. ${ }^{18} \mathrm{Cao}$, et al., ${ }^{16}$ found the serum levels of high-sensitivity C-reactive protein significantly higher in the patients with nonalcoholic steatohepatitis than in the patients with non-alcoholic fatty liver disease. ${ }^{16}$

In some study the role of CRP has been regarded in some autoimmune disorders. ${ }^{19,20}$ In contrast, Yousefi et al studied to compare hsCRP level in Alopecia areata and control group but they did not find any significant difference in two groups. This result was contrary our result. ${ }^{19}$

But recently Coban, et al., ${ }^{21}$ found out a relation between improvement in the Psoriasis Area Severity Index score in psoriasis patients and a significant reduction in high-sensitivity C-reactive protein serum level. ${ }^{21}$ Recently Yang, et al., ${ }^{22}$ showed the level of highsensitivity C-reactive protein can be applied as useful index in early diagnosis of intrauterine bacterial infection..$^{22}$ Mok, et al., ${ }^{23}$ studied the high-sensitivity $\mathrm{C}$-reactive protein level and its relationship with disease activity in systemic lupus erythematosus patients. ${ }^{23}$

High-sensitivity C-reactive protein was detectable in $77 \%$ of systemic lupus erythematosus patients with clinically active disease and correlated with Systemic Lupus Erythematosus Disease Activity Index scores. ${ }^{23}$ In a review article, recently Ridker indicated the serum hsCRP level enhances prognostic data on cardiovascular risk comparable worth to cholesterol or blood pressure. ${ }^{24}$ These are the newest results of studies performed to investigate relation hsCRP level with autoimmune and inflammatory diseases.

Base on these studies, C-reactive protein measures general levels of inflammation. High levels of CRP are caused by infections and many other chronic diseases (including autoimmune and inflammatory diseases). The usage of hsCRP alone is neither sensitive, nor specific in diagnosis of vitiligo but results of our study showed serum hsCRP level might be useful for evaluating the disease activity of vitiligo as Novel biomarker. Maybe combination hsCRP with other markers can reveal more specific associations.

\section{Conclusion}

We found an association between increased serum hsCRP level and generalized vitiligo. The usage of hsCRP alone is neither sensitive, nor specific in diagnosis of vitiligo but this association could imply that hsCRP could intensify the severity of vitiligo thus serum hsCRP level might be useful for evaluating the disease activity of vitiligo as Novel biomarker. Further studies with larger samples and combination hsCRP with other markers are recommended.

\section{Acknowledgements}

We wish to thank Dr. Elaheh Saadatpour without whose help this study could not have been completed. We would also to thank all participating patients for their cooperations, and for their permission to publish this article.

\section{Conflicts of interest}

There are no financial conflicts of interest.

\section{Funding}

None.

\section{References}

1. Kalkanli N, Kalkanli S. Classification and comparative study of vitiligo in Southeast of Turkey with biochemical and immunological parameters. Clin Ter. 2013;164(5):397-402

2. Sheth VM, Gunasekera NS, Silwal S, et al. Development and pilot testing of a vitiligo screening tool. Arch Dermatol Res. 2015;307(1):31-38.

3. Alikhan A, Felsten LM, Daly M, et al. Vitiligo:a comprehensive overview Part I. Introduction, epidemiology, quality of life, diagnosis, differential diagnosis, associations, histopathology, etiology, and workup. J Am Acad Dermatol. 2011;65(3):473-491.

4. Guerra L, Dellambra E, Brescia S, et al. Vitiligo:pathogenetic hypotheses and targets for current therapies. Curr Drug Metab. 2010;11(5):451-467.

5. Caixia T, Hongwen F, Xiran L. Levels of soluble IL-2 receptor in the sera and skin tissue fluids of patients with vitiligo. J Dermatol Sci. 1999;21(1):59-62.

6. Tu CX, Gu JS, Lin XR. Increased interleukin-6 and granulocytemacrophage clony stimulating factor levels in the sera of patients with non-segmental vitiligo. J Dermatol Sci. 2003;31(1):73-78.

7. Sandoval-Cruz M, García-Carrasco M, Sánchez-Porras R, et al. Immunopathogenesis of vitiligo. Autoimmun Rev. 2011;10(12):762-765.

8. Ridker PM, Cushman M, Stampfer MJ. HS CRP in the primary prevention setting. $N$ Engl J Med. 2000;342:836-843.

9. Pearson TA, Mensah GA, Alexander RW, et al. Markers of inflammation and cardiovascular disease:application to clinical and public health practice:A statement for healthcare professionals from the Centers for Disease Control and Prevention and the American Heart Association. Circulation. 2003;107(3):499-511.

10. Miesbach W, Gökpinar B, Gilzinger A. Predictive role of hs-Creactive protein in patients with antiphospholipid syndrome. Immunobiology. 2005;210 (10):755-760.

11. Ridker PM, Danielson E, Fonseca FA. Measuring hsCRP-An Important Part of a Comprehensive Risk Profile or a Clinically Redundant Practice. PLoS Med. 2008;7(2):e1000196.

12. Ramirez D, Patel P, Casillas A. Assessment of high-sensitivity Creactive protein as a marker of airway inflammation in asthma. Ann Allergy Asthma Immunol. 2010;104(6):485-489.

13. Abdou AG, Maraee AH, Reyad W. Immune histochemical expression of heat shock protein 70 in vitiligo. Ann Diagn Pathol. 2013;17(3):245249 .

14. Didem DB, Zafer Y, Çiğdem AD, et al. Serum High Sensitivity C Reactive Protein and Homocysteine Levels in Patients with Mild to Moderate Psoriasis. Turkderm. 2009;43(2):53-57.

15. Kogiso T, Morivoshi Y, Nagahara H. 691 Clinical significance of high sensitivity C-reactive protein (hs-CRP) in non-alcoholic fatty liver disease (NAFLD). Journal of Hepatology. 2006;44(2):S255.

16. Cao Y, Li L. Relationship of non-alcoholic steatohepatitis with arterial endothelial function and atherosclerosis. Zhonghua Gan Zang Bing Za Zhi. 2014;22(3):205-208.

17. Lochhead P, Khalili H, Ananthakrishnan AN, et al. Association Between Circulating Levels of C-Reactive Protein and Interleukin-6 and Risk of Inflammatory Bowel Disease. Clin Gastroenterol Hepatol. 2016;14(6):818-824. 
18. Botta E, Meroño T, Saucedo C, et al. Associations between disease activity, markers of HDL functionality and arterial stiffness in patients with rheumatoid arthritis. Atherosclerosis. 2016;251:438-444.

19. Yousefi M, Namazi MR, Rahimi H, et al. Evaluation of Serum Homocysteine, High-Sensitivity CRP, and RBC Folate in Patients with Alopecia Areata. Indian J Dermatol. 2014;59(6):630.

20. Kumar KV, Priya S, Sharma R, et al. Autoimmune thyroid disease in patients with vitiligo:prevalence study in India. Endocr Pract. 2012;18(2):194-199.

21. Coban M, Tasli L, Turgut S, et al. Association of Adipokines, Insulin Resistance, Hypertension and Dyslipidemia in Patients with Psoriasis Vulgaris. Ann Dermatol. 2016;28(1):74-79.
22. Yang C, Yang Y, Li B, et al. The diagnostic value of high-sensitivity C-reactive protein/albumin ratio in evaluating early-onset infection in premature. Zhonghua Wei Zhong Bing Ji Jiu Yi Xue. 2016;28(2):173177

23. Mok CC, Birmingham DJ, Ho LY, et al. High-sensitivity C-reactive protein, disease activity, and cardiovascular risk factors in systemic lupus erythematosus. Arthritis Care Res (Hoboken). 2013;65(3):441447.

24. Ridker PM. A Test in Context:High-Sensitivity C-reactive protein. $J$ Am Coll Cardiol. 2016;67(6):712-723. 